\title{
Application of acoustic emission technique for bond characterization in FRP-masonry systems
}

\author{
Bahman Ghiassi ${ }^{1, a^{*}}$, Els Verstrynge ${ }^{2, b}$, Paulo B. Lourenço ${ }^{1, c}$ and Daniel V. \\ Oliveira ${ }^{1, d}$ \\ ${ }^{1}$ ISISE, University of Minho, Department of Civil Engineering, Portugal \\ ${ }^{2}$ KU Leuven, Department of Civil Engineering, Building Materials and Technology Division, \\ Belgium

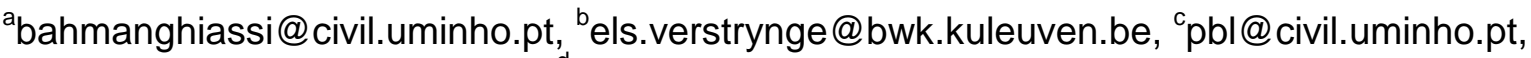 \\ danvco@civil.uminho.pt
}

Keywords: Acoustic emission; FRP; strengthening; masonry; debonding.

\begin{abstract}
The acoustic emission (AE) technique is used for investigating the interfacial fracture and damage propagation in GFRP- and SRG-strengthened bricks during debonding tests. The bond behavior is investigated through single-lap shear bond tests and the fracture progress during the tests is recorded by means of $\mathrm{AE}$ sensors. The fracture progress and active debonding mechanisms are characterized in both specimen types with the aim of AE outputs. Moreover, a clear distinction between the AE outputs of specimens with different failure modes, in both SRG- and GFRPstrengthened specimens, is found which allows characterizing the debonding failure mode based on acoustic emission data.
\end{abstract}

\section{Introduction}

Fiber reinforced materials are frequently used as externally bonded reinforcement for structural enhancement of concrete and masonry structures, such as strengthening of bridges, beams and floor slabs or retrofitting after earthquake damage. Advantages such as low weight to strength ratio and flexibility in application have made these materials interesting. In recent years, composite materials such as fiber reinforced polymers (FRP) and steel reinforced grouts (SRG) have been under investigation for strengthening of (historical) masonry structures. Significant progress has been achieved in the last years regarding experimental and computational investigation of the debonding mechanism and damage in FRP-strengthened masonry elements. Aspects such as compatibility with the masonry substrate and durability of the strengthening solution are under investigation [1-3].

The bond behavior between the strengthening material and the masonry substrate plays an important role in the effectiveness of externally bonded systems. Therefore, investigating and fully characterizing the bond mechanisms is crucial for seismic design and durability assessment of the strengthening solution. Aspects such as failure initiation, interfacial damage propagation and localization, and long-term bond quality monitoring are still under investigation. A comprehensive bond-slip model, for numerical modeling approaches, is also missing for FRP-masonry systems [4]. Development of a health monitoring system for detection and characterization of failure in the strengthened-masonry structures is also of great interest. The latter assist in real time health assessment of historical heritage and application of suitable repair solutions.

This paper aims at investigating how these aspects can be monitored and characterized by means of acoustic emission (AE) technique during experimental testing. A relation between the AE output and bond characteristics such as force-slip behavior, fracture energy, active failure mechanisms and debonding propagation provides valuable information for bond behavior assessment and numerical modeling purposes. Here, the effort is on correlating the failure mode in FRP- and SRGstrengthened brick specimens to the acoustic energies emitted from the specimens during the tests. The AE technique has been extensively used for real time detection of internal damage propagation in structural elements [5,6]. In this technique, piezoelectric sensors are used to detect high- 
frequency mechanical waves produced from the release of strain energy during fracture and crack propagation. It has been found that the $\mathrm{AE}$ outputs are valuable in understanding crack propagation and failure mode in laboratory tests. These findings have also made this technique interesting for on-line structural health monitoring.

\section{Debonding in FRP-masonry}

Failure in FRP-strengthened masonry elements may theoretically occur due to masonry crushing, FRP rupture or FRP debonding from the masonry substrate. The latter is one of the most observed failure modes in experimental tests [1]. FRP deboning initiates from highly stressed regions, near the crack tips or material discontinuities, or from FRP delaminated areas induced due to environmental conditions or poor workmanship. The debonding propagation path depends on the FRP, masonry and FRP-masonry interfacial fracture properties and follows the path with minimum required fracture energy. Therefore, it can occur either through the repair or substrate material or the interface between them.

Debonding in the masonry substrate, denoted as cohesive failure, is the most observed failure mode in the experimental tests [1]. This failure occurs due to the lower mechanical properties of masonry comparing to the repair material and the adhesive. However, depending on the geometrical and material properties and environmental conditions other failure modes may occur. Interfacial debonding, denoted also as adhesive failure, normally occurs in case of poor surface preparation (e.g. when the surface is too smooth or wet upon application of the adhesive). It has also been observed that environmental conditions, especially moist environment, can change the cohesive failure to adhesive failure [2,7]. The tests described in this paper also indicated that specimens subjected to accelerated ageing tests (thermal cycles) are more likely to show adhesive failure. Also a combination of both failure modes has been observed.

In case of strengthening with steel reinforced grout (SRG), in addition to masonry cohesive failure, steel tensile rupture, and adhesive debonding at the mortar-brick interface, debonding at the fibermortar interface may occur. The latter, being the most observed failure modes in the experimental tests performed here, is followed by fibers slipping from the matrix. The typical failure modes in GFRP- and SRG- strengthened brick specimens are presented in Fig. 1.

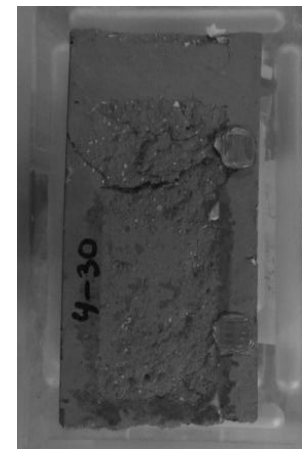

(a)

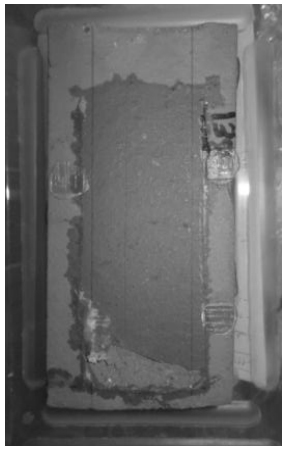

(b)

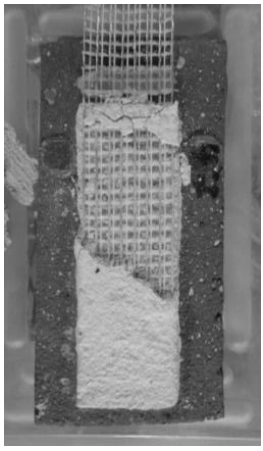

(c)

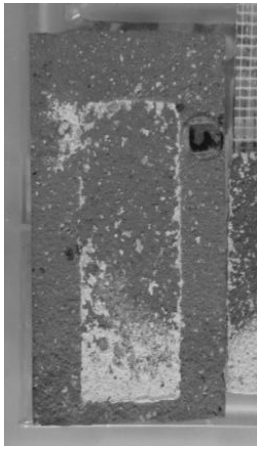

(d)

Fig. 1 Observed failure modes in GFRP- and SRG-strengthened brick specimens: (a) cohesive failure; (b) adhesive failure; (c) steel fibers slipping and mortar cover separation; (d) mortar-brick interface debonding

\section{The Acoustic Emission technique}

Acoustic Emissions (AEs) are high-frequency transient elastic waves that are emitted within the material during local stress redistributions such as micro-crack growth. These emissions are detected on the material's surface by means of piezoelectric transducers, pre-amplified, filtered and 
amplified before being sent to the data logger. The technique has the advantage over other damage detection techniques that it relies on detection of information which is generated by the fracture process itself and allows for on-line damage detection and assessment [8].

A typical AE transient is presented in Fig. 2. Background noise is eliminated through setting a minimum amplitude threshold. An AE hit with a predefined duration is recorded when the threshold is exceeded. For each AE hit, a number of parameters (e.g. arrival time, amplitude, count, duration and energy) and the waveform itself are recorded. The amount of detected AE hits and energy is influenced by the hardware used and software settings, thus software defined parameters (e.g. threshold and sampling frequency) should be kept constant for subsequent tests. The detection of acoustic emissions is also sensitive to a number of setup-specific boundary conditions, such as quality of the coupling between sensor and test specimen, attenuation and speed of wave propagation, source-sensor distance, specimen size and homogeneity.

The recorded acoustic emissions hold information on the fracture process that produced them. Basic $\mathrm{AE}$ hit counting, taking into account the cumulative or average number of AE hits, or emitted $\mathrm{AE}$ energy, has successfully been used for damage assessment in rock, concrete and masonry [5,6]. Other wave properties, such as amplitude or number of threshold crossings (counts) are also used for parameter-based analysis. It is generally observed that micro-cracks generate a large amount of small amplitude emissions, while AE emissions from macro-cracks are fewer but have higher amplitude. Based on this observation, the b-value is applied in seismic analysis to characterize the fracture process by means of the slope of the amplitude distribution. Instead of the seismic b-value, an improved b-value (Ib-value), in which the number of AE data taken into account is set before calculation, is usually applied for AE applications in concrete and rock.

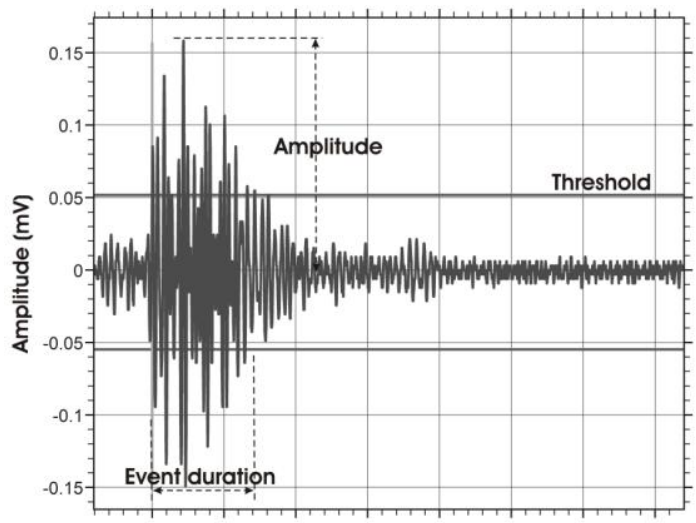

Fig. 2 Typical AE transient event with indication of wave characteristics.

\section{Experimental tests}

The experimental study focuses on detection of the interfacial damage during debonding tests in strengthened masonry bricks by means of the AE technique. The effect of environmental conditions on the bond fracture process and failure mode has also been investigated by performing accelerated ageing tests. This assisted in investigating the differences in fracture properties of the specimens with different failure modes and conditions. Single-lap shear bond tests were performed, before and after environmental exposure, for characterization of the bond behavior. Five specimens from each strengthening type and exposure conditions were tested resulting in total twenty shear bond tests. Among them, twelve specimens were tested with AE, being three reference specimens and three aged specimens for each strengthening type.

Test specimens consisted of bricks strengthened with GFRP and SRG composites. Solid clay bricks with dimensions of 200x100x50 $\mathrm{mm}^{3}$ were used as the substrate. The composite materials were cut with $50 \mathrm{~mm}$ width and applied to the bricks' surface along $150 \mathrm{~mm}$ length of the brick with a 40 mm unbounded part near the loaded end in order to minimize edge effects [1], see Fig. 3. 


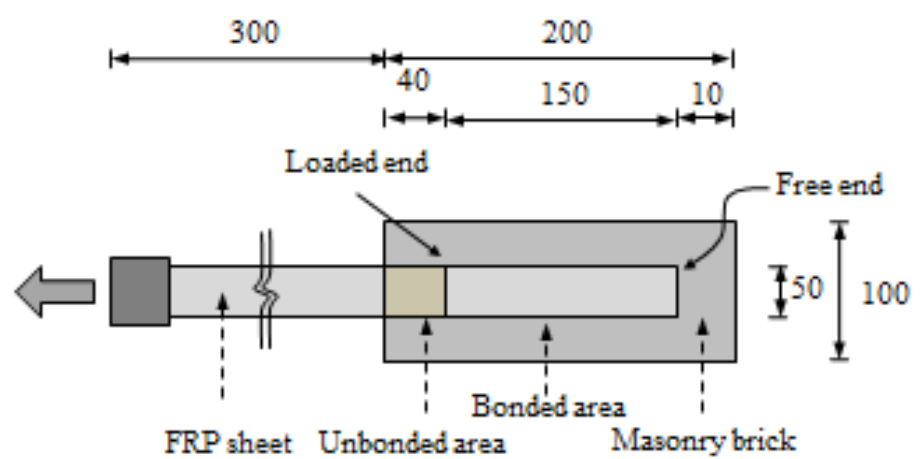

Fig. 3 Geometrical details of the specimens.

In the GFRP-strengthened brick specimens, GFRP strips were applied to the brick surface along its centerline following the wet lay-up procedure, see Fig. 4(a). The bricks were dried in the oven before application of the GFRP sheets. Then, a two-part epoxy primer was applied to the bricks' surfaces for preparation of the substrate surface. Finally, a two-part epoxy resin was used as the matrix for the composite material and adhesion to the masonry substrate.

In SRG-strengthened brick specimens, a medium density steel fiber was used as the reinforcement. The steel fibers were placed on a $3 \mathrm{~mm}$ thick layer of a pozzolanic lime-based (based on an off-theshelf dry mix) mortar which was regularized on the brick's surface. Then, another $3 \mathrm{~mm}$ mortar layer was applied to cover the steel fibers. The bricks' surfaces were sand blasted before application of the SRG in order to increase the mechanical bond between brick and mortar. Although 1 month is proposed in the technical datasheets for complete curing of the mortar, the specimens were cured for three months before performing the tests.

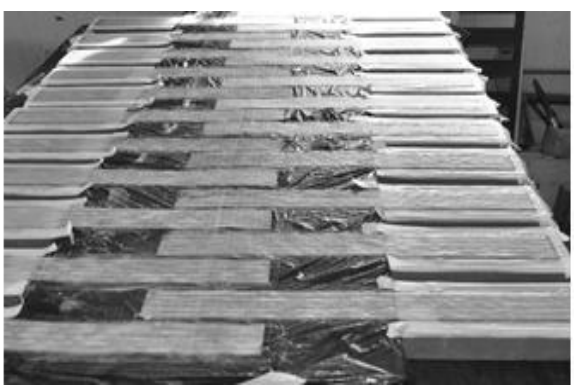

(a)

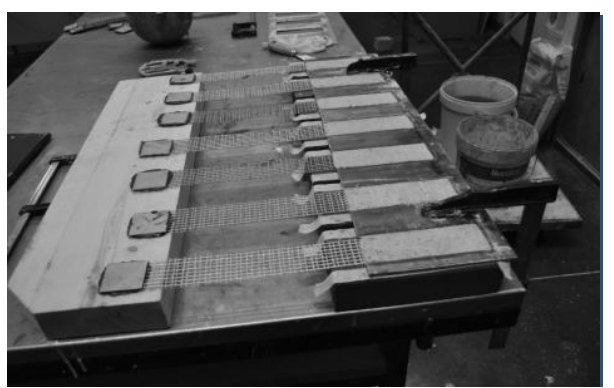

(b)

Fig. 4 (a) GFRP-brick specimens; (b) SRG-brick specimens.

The effect of environmental exposure on the debonding behavior was investigated by performing accelerated ageing tests. The specimens were exposed to 180 cycles (45 days) of hygrothermal conditions in a climatic chamber. The exposure consisted of $6 \mathrm{hr}$ temperature cycles from $+10^{\circ} \mathrm{C}$ to $+50^{\circ} \mathrm{C}$ and constant relative humidity of $60 \%$. In each cycle, the temperature was kept constant at $+10^{\circ} \mathrm{C}$ for $2 \mathrm{hr}$. It was then increased to $+50^{\circ} \mathrm{C}$ in $1 \mathrm{hr}$, followed by $2 \mathrm{hr}$ constant temperature at $+50^{\circ} \mathrm{C}$. Then, the temperature was decreased again to $+10^{\circ} \mathrm{C}$ in $1 \mathrm{hr}$ resulting in $6 \mathrm{hr}$ cycles of exposure. The aim was to investigate the effect of environmental exposure on the debonding fracture properties of the specimens.

\section{Test setup}

Single-lap shear bond tests were performed to investigate the bond behavior in the reference and aged specimens. The tests were performed using a closed-loop servo-controlled testing machine with maximum load capacity of $50 \mathrm{kN}$. A rigid supporting steel frame was used to support the specimens appropriately and avoid misalignments in the load application. The specimens were 
placed on the steel frame and firmly clamped to it as shown in Fig. 5(a). The specimens were pulled monotonically with a rate of $5 \mu \mathrm{m} / \mathrm{sec}$ under displacement control conditions with reference to the LVDT placed at the loaded end of the FRP composite. The resulting load was measured by means of a load cell. The relative slip between the composite material and the brick was measured with the two LVDTs glued at the loaded end and one glued at the free end, see Fig. 5(b).

Acoustic emissions were monitored using a 4-channel Vallen AMSY-5 system with 150-500 kHz operation frequency and $5 \mathrm{MHz}$ sampling rate. Four $150 \mathrm{kHz}$ resonance sensors were attached to opposite sides of the bricks by means of hot melt glue, see Fig.4(b). The preamplifier gain was set to $34 \mathrm{~dB}$ with a fixed threshold level of $40 \mathrm{~dB}$ and pencil lead breaks were used for system calibration. To calculate the AE energy, the AE signal is squared and integrated and the energy unit (eu) is given by $1 \mathrm{eu}=10-14 \mathrm{~V}^{2} \mathrm{~s}$.

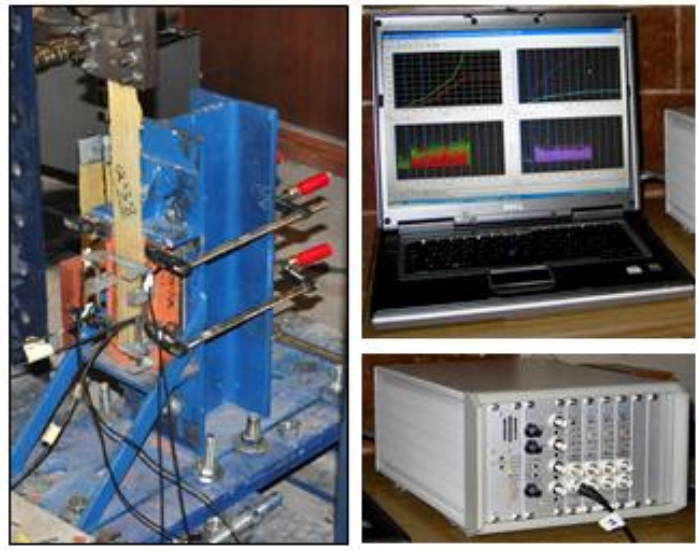

(a)

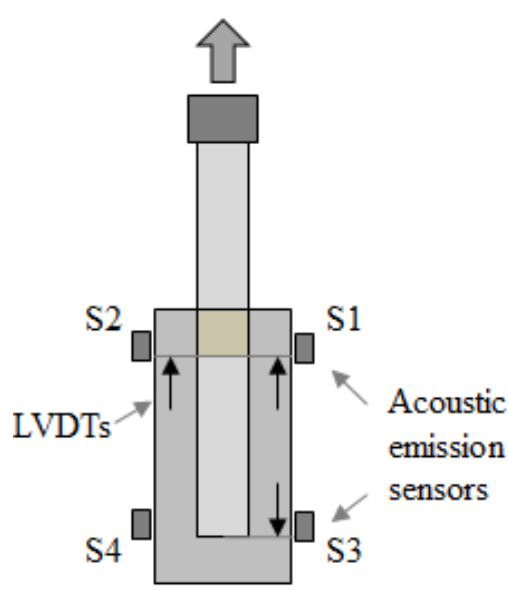

(b)

Fig. 5 (a) Test setup; (b) test instrumentation.

\section{Experimental results}

The summary of the results from the debonding tests is presented in Table 1 in terms of the average maximum debonding force. A slight $6 \%$ reduction of the average maximum debonding force was observed in GFRP-strengthened brick specimens after exposure to environmental conditions, which contrasts with a significant $27 \%$ reduction for SRG-strengthened brick specimens. In terms of failure mode, a change from cohesive failure to adhesive failure after exposure to accelerated tests was observed in the GFRP-brick specimens. The observed failure modes in the reference specimens were cohesive failure with fracture inside the brick (1 specimen) or a combination of cohesive/adhesive failure ( 2 specimens). The cohesive fracture occurred in a relatively deep layer of the brick (around $10 \mathrm{~mm}$ ). On the other hand, the failure mode in the aged specimens was predominantly adhesive. The fracture surface was at the FRP/brick interface in all three specimens. The adhesive failure was combined with detachment of a brick bulb at the free end in two specimens. In SRG-strengthened brick specimens, two failure modes were observed: (a) steel fibers slipping and mortar cover separation, which was the main observed failure mode and (b) detachment of mortar from the brick's surface, which happened only in one reference specimen.

Table 1. Average debonding force of the tested specimens.

\begin{tabular}{cccc}
\hline Specimens & Condition & $\begin{array}{c}P_{\max } \\
(\mathrm{kN})\end{array}$ & $\begin{array}{c}\mathrm{CoV} \\
(\%)\end{array}$ \\
\hline \multirow{2}{*}{ GFRP-brick } & Reference & 10.0 & 5.3 \\
\cline { 2 - 4 } & Aged & 9.4 & 20.6 \\
\hline \multirow{2}{*}{ SRG-brick } & Reference & 4.1 & 14.4 \\
\cline { 2 - 4 } & Aged & 3.0 & 20.0 \\
\hline
\end{tabular}


Typical AE results obtained from the debonding tests are shown in Figs 6 for a GFRP-brick specimen. It can be seen that as the debonding progresses, the amount of emitted AE energies increase. The final peak in the AE energy curve, Fig. 6(a), is corresponding to the delamination moment which has occurred with releasing of a high amount of energy.

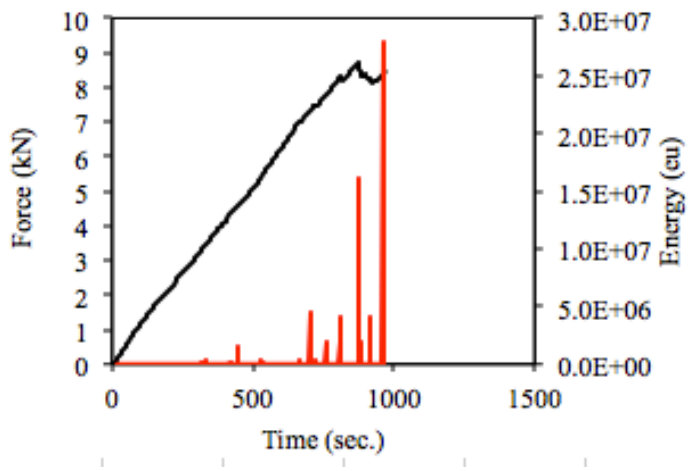

(a)

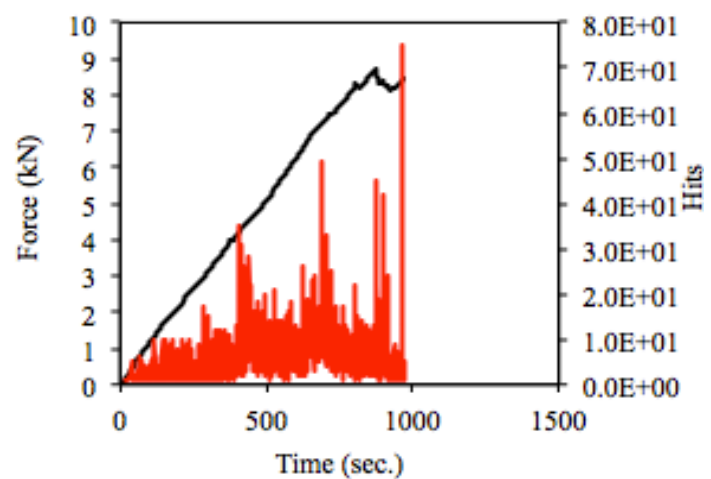

(b)

Fig. 6 AE output from debonding tests on a GFRP-brick specimen: (a) AE energy; (b) AE hits.

A comparison is made next between the AE outputs in specimens with different failure modes. The aim was to investigate the existence of any correlation between the fracture process and the $\mathrm{AE}$ outputs. In GFRP-brick specimens, the comparison is made between cohesive and adhesive failure modes, see Figs. 7, 8. It can be observed that in the specimen with cohesive debonding the AE energy remains relatively low throughout the test, accompanied by a sudden and large amount of $\mathrm{AE}$ energy release when the debonding occurs at the end of the test, Fig. 7. The observed behavior confirms the brittle and sudden nature of the cohesive debonding. On the contrary, in the specimen with cohesive/adhesive failure a progressive release of energy is observed during the test. The high rate of energy detection shows the high number of active cracks and progressive failure during the tests. Progressive detection of AE energies is observed until the complete debonding. However, the magnitude of the detected energies is much lower than the ones detected in the specimens with cohesive failure mode. This large difference is due to the different nature and fracture properties of brick and FRP/brick interface. The specimen with adhesive failure combined with formation of a brick bulb at the free end shows a similar AE energy emission to the specimen with pure adhesive failure. However, a large amount of energy is released in this specimen before debonding due to the brick bulb fracture at the free end. Fig. 8 presents the energy/hit $(\mathrm{E} / \mathrm{h})$ ratio during the tests. The cohesive failure shows a peak at the end of the tests showing that a strong energy has been emitted at the moment of debonding. However, several peaks can be observed in the specimens with adhesive debonding showing the progressive nature of failure in this mode.

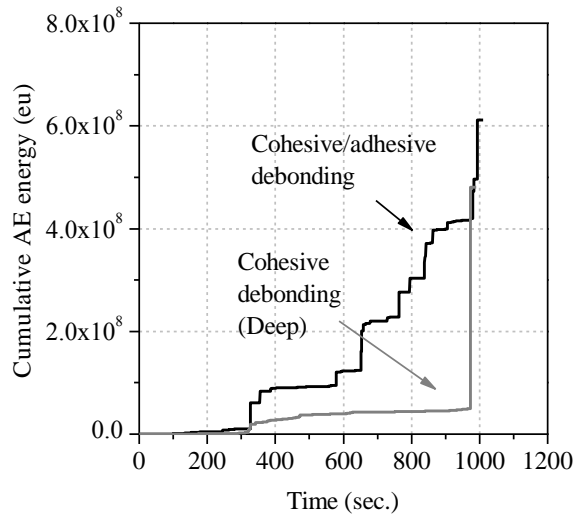

(a)

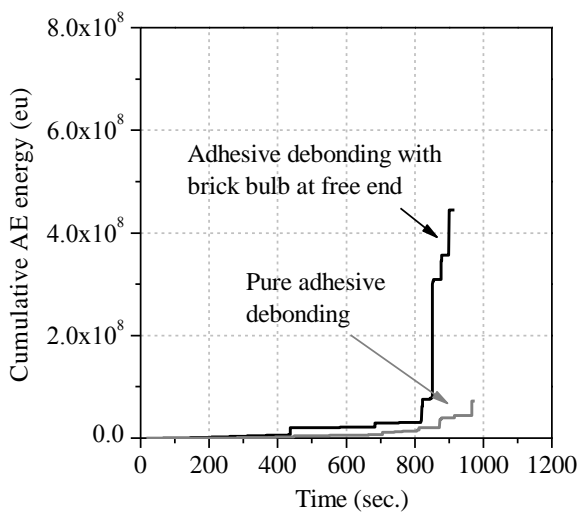

(b)

Fig. 7 Comparison of different failure modes in GFRP-strengthened bricks: (a) cohesive debonding; (b) adhesive debonding. 


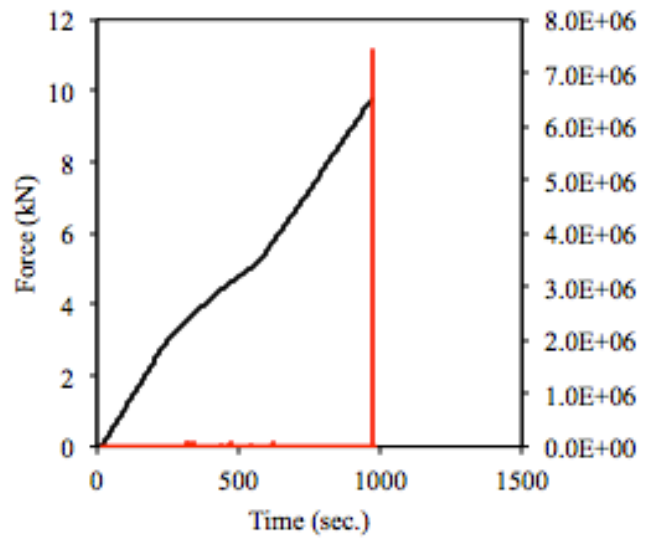

(a)

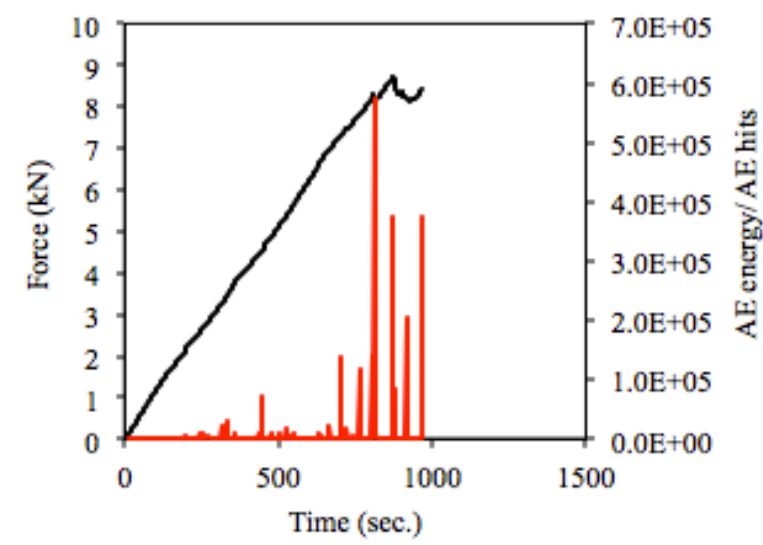

(b)

Fig. $8 \mathrm{E} / \mathrm{h}$ ratio in GFRP-strengthened bricks for different failure modes: (a) cohesive debonding;

(b) adhesive debonding.

Fig. 9 presents a comparison between two failure modes in SRG-brick specimens. A brittle behavior is observed in the specimen with brick/mortar detachment failure. The detected energy in this specimen is very low during the test followed by a sudden release of energy at the moment of debonding. On the other hand, the fibers slipping failure mode produces a progressive release of energy during the test until diminishing the adhesive bond, followed by a reduction of the AE energy rate in the stage governed by frictional resistance. This E/h curve, Fig. 10, confirms this hypothesis. Again, in the specimens with mortar detachment failure a large peak is observed at the moment of debonding, while the specimen with fiber slipping failure shows several peak during the test.

The results shows that the failure mode can be satisfactorily distinguished from the AE outputs. Further research is required in understanding the correlation between the AE output and fracture parameters such as fracture energy or bond-slip behavior.

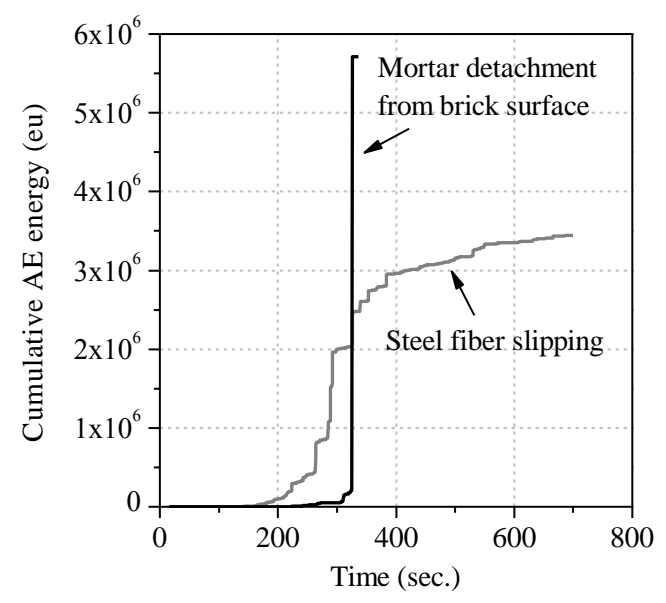

Fig. 9 Comparison of different failure modes in SRG-strengthened bricks. 


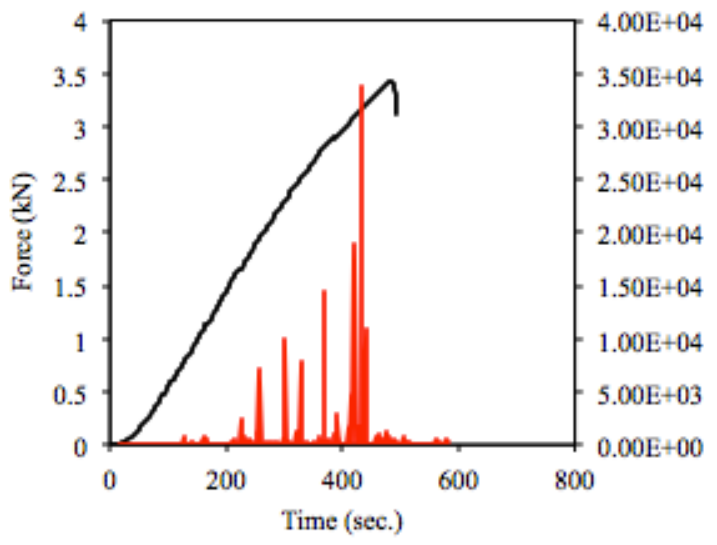

(a)

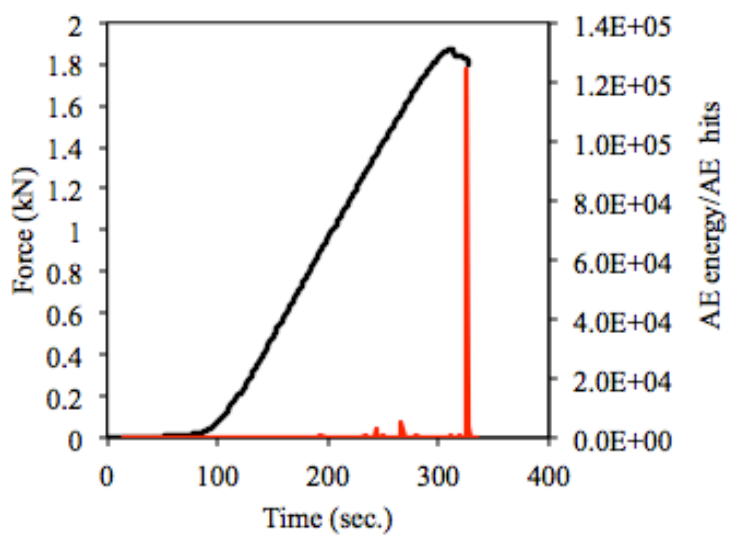

(b)

Fig. 10 E/h ratio in SRG-strengthened bricks for different failure modes: (a) steel fiber slipping; (b) mortar detachment.

\section{Conclusions}

The AE technique was applied to detect and characterize the failure mode during the debonding of composite materials from masonry bricks. The specimens consisted of solid clay bricks strengthened with GFRP and SRG composites. The debonding phenomenon was investigated by performing single-lap shear bond tests on the specimens. In order to obtain different values of bond strength and failure mode, accelerated ageing tests were also carried out. The failure mode of the GFRP-strengthened specimens changed after exposure from cohesive to adhesive failure which assisted in evaluating the AE applicability in detecting different failure modes. This can be helpful in interpretation of structure condition during laboratorial testing or onsite health monitoring.

The AE data, and more specifically the detected AE energy, was found to be in correlation with the failure mode in both type of specimens. However, further experimental tests are required for a better understanding of the advantages of the technique and establishing correlations between fracture properties an AE outputs is necessary.

\section{References}

[1] Valluzzi MR, Oliveira D V, Caratelli A, Castori G, Corradi M, Felice G, et al. Round Robin Test for composite-to-brick shear bond characterization. Mater Struct 2012;45:1761-91.

[2] Ghiassi B, Oliveira D V, Lourenço PB. Recent development in durability of FRP-masonry systems. Int. Conf. Rehabil. Restor. Struct. , India: 2013.

[3] Ghiassi B, Marcari G, Oliveira D V, Lourenço PB. Water degrading effects on the bond behavior in FRP-strengthened masonry. Compos Part B Eng 2013;54:11-9.

[4] Ghiassi B, Marcari G, Oliveira DV, Lourenço PB. Numerical analysis of bond behavior between masonry bricks and composite materials. Eng Struct 2012;43:210-20.

[5] Grosse CU, Ohtsu M. Acoustic emission testing - basics for research - applications in civil engineering. Springer; 2008.

[6] Tomor A, Verstrynge E. A joint fatigue - creep deterioration model for masonry with acoustic emission based damage assessment. Constr Build Mater. Constr Build Mater 2013;43:57588.

[7] Ghiassi B, Oliveira DV, Lourenço PB. Hygrothermal durability of bond in FRP-strengthened maosnry. Mater Struct n.d.

[8] Wevers M. Listening to the sound of materials: Acoustic emission for the analysis of material behavior. NDT E Int 1997;30:99-106. 